\title{
RÉPTEIS DO CAMPUS DA UNIVERSIDADE FEDERAL DE SANTA MARIA, RS, BRASIL
}

Tiago Gomes dos Santos*, Katia Alcione Kopp, Marcia Regina Spies, Rafael Trevisan, Sonia Zanini Cechin

Biota Neotropica v5 (n1) - http://www.biotaneotropica.org.br/v5n1/pt/abstract?inventory+BN02705012005

Recebido: 06/04/2004

Revisado: $17 / 12 / 2004$

Publicado: 01/01/2005

Departamento de Biologia, Centro de Ciências Naturais e Exatas, Universidade Federal de Santa Maria (www.ufsm.br), Av. Roraima s/ n, 97105-900, Santa Maria, RS, Brasil.

*frogomes@yahoo.com.br

\begin{abstract}
This work presents an inventory of reptile species occuring in the campus of Universidade Federal de Santa Maria (UFSM) and surroundings. It is based on monthly field expeditions between November 2001 and October 2002 and examination of material in the Coleção Herpetológica do Departamento de Biologia da UFSM. Of the 36 species listed, 20 were recorded in the field whereas 16 were recorded in the collection.
\end{abstract}

Key words: Reptiles, inventory, Universidade Federal de Santa Maria, Santa Maria.

\section{Resumo}

Este trabalho apresenta um inventário das espécies de répteis que ocorrem no campus da Universidade Federal de Santa Maria (UFSM) e arredores. A listagem está baseada em expedições mensais de campo entre novembro de 2001 e outubro de 2002 e exame do material da Coleção Herpetológica do Departamento de Biologia da UFSM. Das 36 espécies listadas, 20 foram registradas no campo e 16 foram registradas pelo exame da coleção.

Palavras-chave: Répteis, inventário, Universidade Federal de Santa Maria, Santa Maria. 


\section{INTRODUÇÃO}

Estudos sobre composição faunística são fundamentais para a compreensão da biodiversidade e conseqüentemente para o planejamento e tomada de decisões sobre estratégias de conservação (Haddad, 1998). Existem apenas dois estudos de listagem dos répteis abrangendo todo o Estado do Rio Grande do Sul, (Lema 1994; 2002), embora os primeiros levantamentos de répteis nesse estado datem do século XIX (Boulenger, 1886).

Listas de espécies para algumas regiões do Estado são disponíveis (e.g. Lema \& Fabián-Beurmann, 1977; Lema et al., 1980; Gomes \& Krause, 1982; Lema et al., 1984; Cechin, 2002). Lema \& Ferreira (1990) publicaram uma lista sistemática comentada dos testudines do Rio Grande do Sul e afirmaram que a fauna conhecida de quelônios é pobre em número de espécies, mas apresenta ainda problemas pela escassez de material de coleções e de especialistas.

Algumas regiões foram pouco estudadas, como por exemplo, as áreas pertencentes aos Campos Sulinos (Haddad \& Abe, 2000). Na Depressão Central, situada no centro do Estado do Rio Grande do Sul, há registro de 25 espécies de serpentes para uma área de 5.786 hectares, estudada no município de Santa Maria (S.T.Z Cechin, observação pessoal), recoberta em aproximadamente $30 \%$ por floresta nativa densa (Floresta Estacional Decidual) e o restante por campo nativo (Pereira et al., 1989).

Em um segundo estudo abrangendo municípios situados na Encosta Inferior do Nordeste e Depressão Central do Estado, Cechin (2002) registrou 42 espécies de répteis, das quais 16 são exclusivas de áreas de campo, oito são exclusivas de mata e 18 podem ocupar ambos os tipos de formações. Neste último estudo, compreendendo nove municípios, oito pertencem à região de Floresta Estacional Decidual e apenas um aos Campos Sulinos.

Muitos estudos de répteis são realizados em áreas relativamente conservadas (e.g. Gomes \& Krause, 1982; Martins \& Oliveira, 1999; Marques \& Sazima, 2004), existindo poucos dados sobre áreas sob intensa ação antrópica (Lema et al., 1980; 1984). O presente estudo visou realizar o inventário de répteis em área alterada no campus da Universidade Federal de Santa Maria e comparar a fauna nos diferentes ambientes amostrados.

\section{MATERIAL E MÉTOdOS}

O campus da Universidade Federal de Santa Maria (UFSM) encontra-se a uma altitude de aproximadamente 95 $\mathrm{m}$, na região fisiográfica da Depressão Central (2942'S; $\left.53^{\circ} 42^{\prime} \mathrm{W}\right)$, no bioma dos Campos Sulinos, RS, e está a aproximadamente $10 \mathrm{~km}$ do rebordo sul do Planalto Meridional Brasileiro (fig.1).

A área de amostragem possui aproximadamente $6 \mathrm{~km}^{2}$ e os diferentes ambientes nela encontrados apresentam-se bastante fragmentados e distribuídos irregularmente (fig. 2). Aproximadamente 35\% são urbanizados, 30\% são recobertos por campo nativo, $20 \%$ por lavouras (e.g. soja, milho, sorgo, horticultura, pastagens) e os $15 \%$ restantes são ocupados por capoeira, fragmentos de silvicultura de espécies exóticas (Eucalyptus e Pinus) e fragmentos de mata secundária em regeneração, com predomínio de Prunus sellowii Koehne (Rosaceae).

De acordo com a classificação de Köppen, o município de Santa Maria apresenta clima temperado chuvoso e quente do tipo Cfa. As precipitações são regulares ao longo do ano, com índices pluviométricos anuais de 1500 a $1750 \mathrm{~mm}$ (Pereira et al., 1989).

Os répteis foram amostrados de novembro de 2001 a outubro de 2002, pelo método de procura limitada por tempo, com esforço de três coletores. A área de estudo foi dividida em quatro subáreas, compreendendo os seguintes ambientes: A1 (zona urbana, margens de corpos d'água, mata e capoeira); A2 (lavoura, capoeira, margens de corpos d'água e banhado); A3 (capoeira, mata e margens de corpos d'água); A4 (campo nativo, lavoura, margens de corpos d'água e banhado). Cada subárea foi amostrada mensalmente, por três horas no período diurno e três horas no período noturno. Os animais encontrados vivos foram capturados manualmente, fotografados e soltos após a identificação. Os animais encontrados mortos foram incorporados à Coleção Científica da UFSM.

Para complementar a listagem, foram incorporados os dados da Coleção Científica (Departamento de Biologia, Setor de Zoologia, ZUFSM) da UFSM, considerando os exemplares coletados no campus e área limítrofe, num raio de $6 \mathrm{~km}$ do campus, com fisionomia semelhante à área campestre presente no campus.

A identificação dos répteis foi efetuada com base em Peters \& Donoso-Barros (1970), Peters \& Orejas-Miranda (1970), Vanzolini (1986, 2002), Dixon (1989) e Lema (1994, 2002).

Uma curva do coletor média foi construída a partir de 50 adições aleatórias das amostras, utilizando o programa EstimateS 7.0 (Colwell, 2004).

A similaridade faunística da área de estudo foi comparada com outras três áreas estudadas no Rio Grande do Sul (Gomes \& Krause, 1982; Lema et al.,1980, 1984), utilizando o Coeficiente de Similaridade Binário de Sorensen (Krebs, 1999):

$$
\mathrm{Ss}=\mathbf{2 A} /(\mathbf{2 A}+\mathbf{B}+\mathrm{C})
$$

Onde: A é o número de espécies comuns a ambas as áreas; B é o número de espécies presentes na área 1, mas ausentes na área 2; C é o número de espécies presentes na área 2, mas ausentes na área 1 . O índice varia de 0 (dissimilaridade máxima) a 1 (similaridade máxima). 


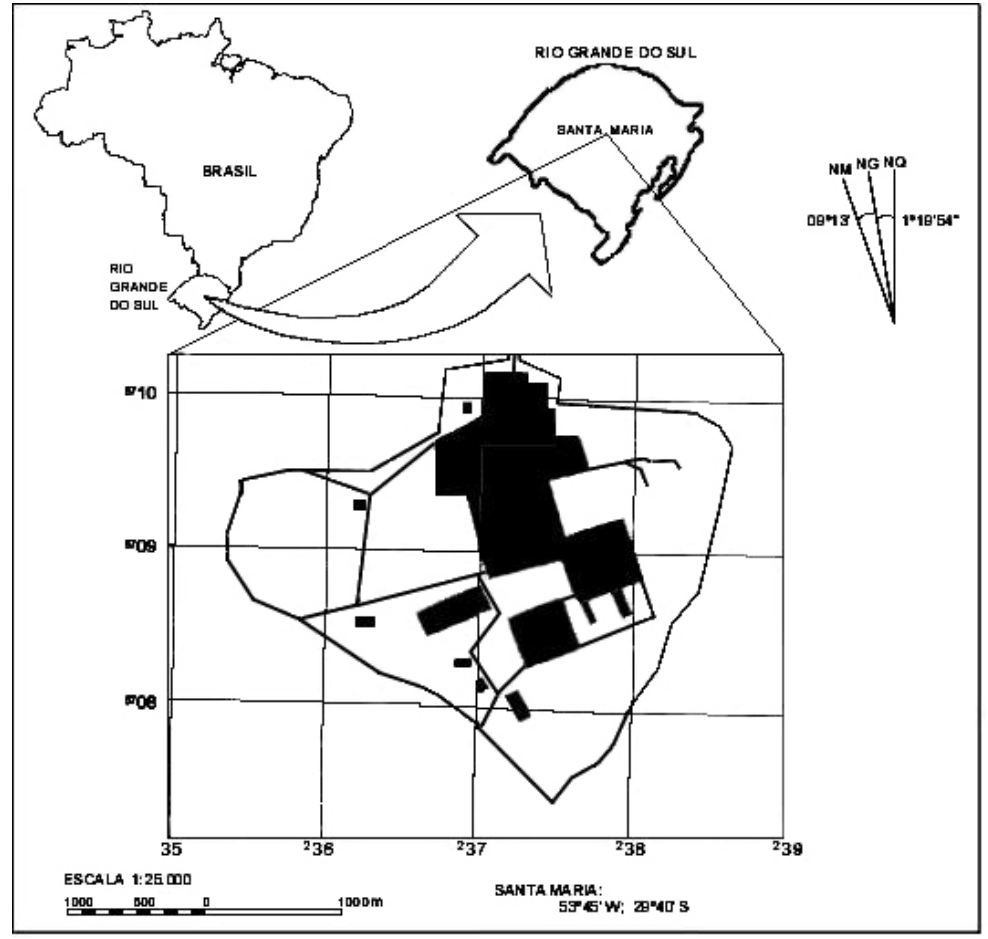

Figura 1. Mapa da localização do Campus da Universidade federal de Santa Maria (UFSM), destacando a porção urbanizada da área (em preto).

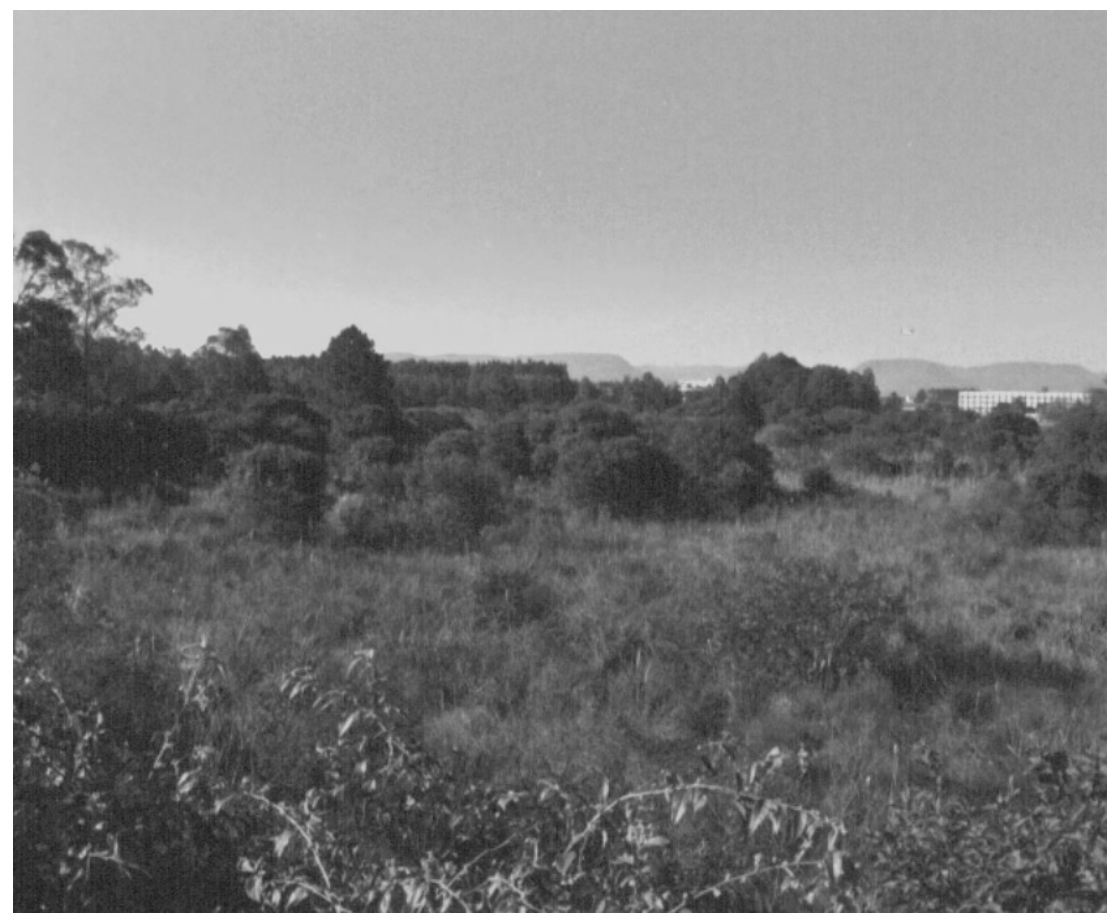

Figura 2. Foto da fitofisionomia do campus Universidade Federal de Santa Maria/RS. 


\section{RESULTADOS E DISCUSSÃO}

Em doze campanhas, totalizando 288 horas/ observador de procura, foram identificadas na área do campus da UFSM 20 espécies de répteis, distribuídas em doze famílias. O exame do material da Coleção Herpetológica da UFSM, depositado ao longo de vários anos, oriundo do perímetro da área de estudo (raio de $6 \mathrm{~km}$ ) possibilitou acrescentar outras 16 espécies ao presente inventário (Tabela I).

A curva cumulativa média de espécies obtida ao final de 12 campanhas não atingiu a assíntota (fig. 3), demonstrando que a riqueza na área é potencialmente maior que a registrada. Assim, para ampliar o número de táxons registrados seria necessário um esforço amostral maior, associado com o uso de métodos adicionais (e.g. pitfall-trap e coleta com auxílio de terceiros). No entanto, o exame da Coleção Herpetológica da Instituição, depositária de exemplares da região de estudo ao longo de 15 anos, possibilitou a confecção de uma lista de espécies mais completa.

A família com maior número de representantes foi Colubridae, com 21 espécies, totalizando 58,3\% dos répteis amostrados (fig. 4). Entre os colubrídeos, 90,5\% $(\mathrm{n}=19)$ pertencem à subfamília Xenodontinae e 9,5\% $(\mathrm{n}=2)$ à Colubrinae, o que é proporcional à representatividade na América do Sul: Xenodontinae possui 60 gêneros, ao passo que, Colubrinae está representada por apenas 12 gêneros (Duellman, 1979).

A maioria das espécies registradas $(n=18 ; 51,4 \%)$ são comumente encontradas em áreas campestres do Rio Grande do Sul, seguidas por aquelas que podem ocupar desde áreas florestadas até campos $(\mathrm{n}=15 ; 42,9 \%)$, Tabela I (sensu Lema et al., 1984 e Lema, 1994). Apenas duas espécies possuem distribuição restrita a áreas florestadas, Tabela I. Aproximadamente $26 \%$ das espécies $(n=9)$ são reconhecidamente associadas a hábitats alterados do Estado, como áreas peridomiciliares e lavouras, Tabela I (sensu Lema et al., 1984 e Lema, 1994). Hemidactylus mabouia não foi considerada nestas relações por se tratar de espécie exótica (Lema, 1994).

A ocorrência das espécies nos diversos ambientes estudados mostra que a maior riqueza foi encontrada no campo $(n=25$ espécies $)$ e na área urbana $(n=23$ espécies $)$, respectivamente. Entretanto, a maioria das espécies (66\%) não foi exclusiva de um único ambiente (Tabela I). Isso sugere um baixo grau de especialização dessa herpetofauna quanto ao uso de hábitat.

O maior número de registros efetuados em área de campo está relacionado à grande representatividade deste ambiente na fitofisionomia da área de estudos e arredores. Todos os espécimes registrados na área urbana (exceto Tupinambis merianae, Hemidactylus mabouia, Sibynomorphus ventrimaculatus e Liophis poecilogyrus) foram encontrados mortos por atropelamento, o que pode indicar fluxo da herpetofauana entre fragmentos de habitat na área de estudo e evidencia o impacto da urbanização sobre a fauna de répteis local.

A riqueza de espécies do presente estudo é comparada com três inventários de répteis desenvolvidos no Rio Grande do Sul (Lema et al., 1980; Gomes \& Krause, 1982; Lema et al., 1984), pela aplicação do Coeficiente de Similaridade de Sorensen (Ss) (Tabela II).

Quanto à similaridade faunística (Tabela II), o maior valor foi encontrado entre o campus da UFSM e a região da grande Porto Alegre $(\mathrm{Ss}=0,69)$ e norte da grande Porto Alegre $\left(\mathrm{Ss}_{\mathrm{s}}=0,63\right)$, estudadas por Lema et al. $(1980,1984)$, respectivamente. Essa maior similaridade pode estar relacionada ao fato das três áreas situarem-se, em grande parte ou totalmente, na Depressão Central e próximas ao rebordo do Planalto Meridional Brasileiro. De acordo com Lema (1971), ocorre dispersão de fauna entre o Planalto e a Depressão Central do Estado. Essa característica transicional da herpetofauna foi encontrada na Grande Porto Alegre e norte da Grande Porto Alegre por Lema et al. (1980, 1984, respectivamente) onde, do ponto de vista zoogeográfico, a fauna de répteis é composta por elementos do planalto (Província Zoogeográfica Guarani e em menor influência, a Província Tupi) e elementos da campanha (Província Zoogeográfica Pampeana), mas com predomínio de espécies da savana, a qual se estende do Rio Grande do Sul a países limítrofes.

A fauna do campus da UFSM apresentou menor similaridade $(\mathrm{Ss}=0,49)$ com a fauna da Estação Ecológica do Taim (Gomes \& Krause, 1982) (Tabela II). Essa menor similaridade provavelmente decorre de diferenças fitofisionômicas pois, apesar de apresentar campos e capões de mata comuns à Depressão Central, a Estação Ecológica do Taim está situada na faixa litorânea do Estado e abrange grandes extensões de dunas sem vegetação e banhados (Gomes \& Krause, 1982) que, segundo Lema et al. (1994), recebem elementos herpetofaunísticos do litoral uruguaio.

No último levantamento de répteis do Rio Grande do Sul, Lema (1994) listou 113 espécies, sendo que no presente estudo registraram-se 36 , o que corresponde a $32 \%$ dos répteis do Estado. Esse percentual pode ser considerado alto, tendo em vista a pequena extensão da área aqui estudada $\left(6 \mathrm{~km}^{2}\right)$ em relação à área total do Estado (282.062 $\mathrm{km}^{2}$ ).

Todas as espécies encontradas no campus da UFSM já haviam sido registradas para o Estado, mas é importante destacar o reencontro de Cercosaura ocellata petersi em área de campo, a cerca de $6 \mathrm{~km}$ da área central de estudo. Dois espécimes desse sáurio foram coletados no RS (município de Santa Maria) apenas em 1952, data da descrição da subespécie (Ruibal, 1952). 


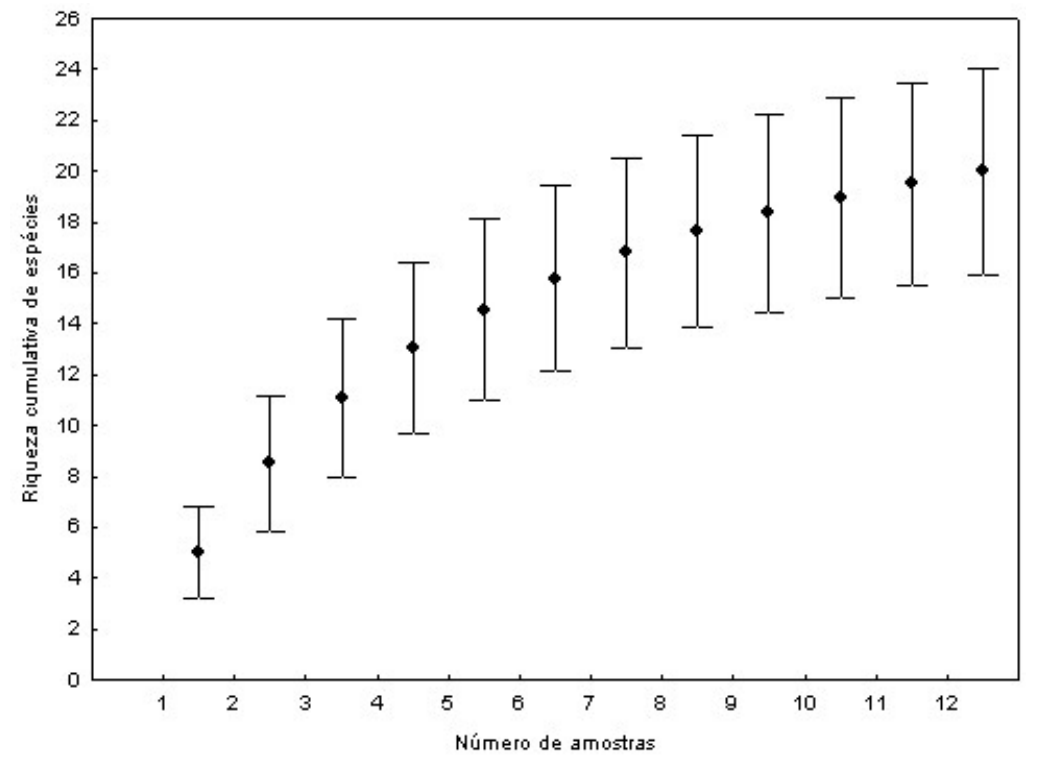

Figura 3. Curva cumulativa para 20 espécies de répteis registradas no campus da Universidade Federal de Santa Maria/RS entre novembro de 2001 e outubro de 2002. Os pontos expressam a curva cumulativa média, gerada pela adição aleatória das amostras, e as barras verticais indicam o desvio padrão em torno da curva média.



Figura 4. Contribuição relativa das familias na composição da fauna de répteis da Universidade Federal de Santa Maria, $R S$. 
Santos, T.G.; Kopp, K. A.; Spies, M.R.; Trevisan, R. \& Cechin, S. Z. - Biota Neotropica, v5 (n1) - BN02705012005

Tabela I - Listagem dos répteis do Campus da UFSM, RS. Registro: $C$ = espécies encontradas no campus da UFSM entre novembro de 2001 e outubro de 2002; ZUFSM = registros da Coleção Herpetológica da UFSM; Hábitat: segundo Lema et al. (1984) e Lema (1994). Ambientes onde as espécies foram registradas: $C A=$ campo; $C P=$ capoeira; $M=$ mata; $M A=$ margens de corpos d'água $L=$ lavoura; $A U=$ área urbana

\begin{tabular}{|c|c|c|c|}
\hline Família/Espécie & Registro & Hábitat & Ambientes \\
\hline \multicolumn{4}{|l|}{ TESTUDINES } \\
\hline \multicolumn{4}{|l|}{ Emydidae } \\
\hline Trachemys dorbigni ( Duméril \& Bibron, 1835) & $\mathrm{C}$ & Campo & MA, B \\
\hline \multicolumn{4}{|l|}{ Chelidae } \\
\hline Hydromedusa tectifera Cope, 1870 & $\mathrm{C}$ & Campo & $\mathrm{MA}, \mathrm{AU}$ \\
\hline \multicolumn{4}{|l|}{ SAURIA } \\
\hline \multicolumn{4}{|l|}{ Gekkonidae } \\
\hline Hemidactylus mabouia (Moreau de Jonnès, 1818) & $\mathrm{C}$ & Espécie exótica & $\mathrm{AU}$ \\
\hline \multicolumn{4}{|l|}{ Gymnophtalmidae } \\
\hline Cercosaura ocellata petersi Ruibal, 1952 & ZUFSM & Mata e campo & $\mathrm{CA}$ \\
\hline Pantodactylus schreibersii (Wiegmann, 1834) & ZUFSM & Campo & CA \\
\hline \multicolumn{4}{|l|}{ Teiidae } \\
\hline Teius oculatus (d'Órbigny \& Bibron, 1837) & $\mathrm{C}$ & Campo & $\mathrm{CA}, \mathrm{AU}$ \\
\hline Tupinambis merianae (Dumeril \& Bibron, 1839) & $\mathrm{C}$ & Mata e campo & $\mathrm{CP}, \mathrm{MA}, \mathrm{AU}$ \\
\hline \multicolumn{4}{|l|}{ Scincidae } \\
\hline Mabuya dorsivittata Cope, 1862 & $\mathrm{C}$ & Campo & CA \\
\hline \multicolumn{4}{|l|}{ Anguidae } \\
\hline Ophiodes sp. Wagler, 1828 & ZUFSM & Campo & $\mathrm{CA}$ \\
\hline \multicolumn{4}{|l|}{ AMPHISBAENIA } \\
\hline \multicolumn{4}{|l|}{ Amphisbaenidae } \\
\hline Amphisbaena munoai Klappenbach, 1960 & ZUFSM & Campo & CA \\
\hline Anops kingii Bell, 1833 & ZUFSM & Campo & $\mathrm{AU}$ \\
\hline \multicolumn{4}{|l|}{ SERPENTES } \\
\hline \multicolumn{4}{|l|}{ Colubridae } \\
\hline \multicolumn{4}{|l|}{ Colubrinae } \\
\hline Mastigodryas bifossatus (Raddi, 1820) & $\mathrm{C}$ & Mata e campo & $\mathrm{CA}, \mathrm{AU}$ \\
\hline Tantilla melanocephala (Linnaeus, 1758) & ZUFSM & Campo e peridomiciliar & CA \\
\hline \multicolumn{4}{|l|}{ Xenodontinae } \\
\hline Atractus reticulatus (Boulenger, 1885) & ZUFSM & Mata e campo & $\mathrm{CA}, \mathrm{AU}$ \\
\hline Boiruna maculata (Boulenger, 1896) & ZUFSM & Mata e campo & $\mathrm{CA}, \mathrm{AU}$ \\
\hline Echinantera cf. occipitalis (Jan, 1863) & ZUFSM & Campo & $\mathrm{CA}$ \\
\hline Helicops infrataeniatus Jan, 1865 & $\mathrm{C}$ & Mata e campo & MA, AU \\
\hline Liophis anomalus (Günther, 1858) & ZUFSM & Campo & AU \\
\hline Liophis flavifrenatus (Cope, 1862) & ZUFSM & Campo & $\mathrm{CA}$ \\
\hline Liophis jaegeri (Günther, 1858 ) & $\mathrm{C}$ & Campo & $\mathrm{CA}, \mathrm{B}, \mathrm{AU}$ \\
\hline Liophis miliaris (Linnaeus, 1858) & $\mathrm{C}$ & Mata e campo & $\mathrm{CA}, \mathrm{MA}, \mathrm{AU}$ \\
\hline Liophis poecilogyrus (Wied, 1825) & $\mathrm{C}$ & Mata, campo e peridomiciliar & $\mathrm{CA}, \mathrm{AU}$ \\
\hline Lystrophis dorbignyi (Duméril, Bibron \& Duméril, 1854) & $\mathrm{C}$ & Campo e peridomiciliar & $\mathrm{CA}, \mathrm{AU}$ \\
\hline Oxyrhopus rhombifer Duméril, Bibron \& Duméril, 1854 & $\mathrm{C}$ & Mata e campo & $\mathrm{CA}, \mathrm{M}, \mathrm{AU}$ \\
\hline Philodryas aestivus (Duméril, Bribon \& Duméril, 1854) & $\mathrm{C}$ & Mata e peridomiciliar & $\mathrm{CA}, \mathrm{CP}, \mathrm{AU}$ \\
\hline Philodryas patagoniensis (Girard, 1858) & ZUFSM & Campo e peridomiciliar & $\mathrm{CA}, \mathrm{AU}$ \\
\hline Philodryas olfersii (Lichtenstein, 1823) & ZUFSM & Mata e peridomiciliar & $\mathrm{AU}$ \\
\hline Pseudablabes agassizii (Jan, 1863) & ZUFSM & Campo & $\mathrm{CA}$ \\
\hline Sibynomorphus ventrimaculatus (Boulenger, 1885) & $\mathrm{C}$ & Campo e peridomiciliar & $\mathrm{CA}, \mathrm{AU}$ \\
\hline Thamnodynastes strigatus (Güenther, 1858) & ZUFSM & Mata e campo & MA \\
\hline Thamnodynastes hypoconia (Cope, 1860$)$ & $\mathrm{C}$ & Mata e campo & $\mathrm{CA}, \mathrm{L}, \mathrm{AU}$ \\
\hline Waglerophis merremii (Wagler, 1824) & $\mathrm{C}$ & Mata e campo & $\mathrm{CA}, \mathrm{AU}$ \\
\hline \multicolumn{4}{|l|}{ Elapidae } \\
\hline Micrurus altirostris (Cope, 1860) & $\mathrm{C}$ & Mata, campo e peridomiciliar & $\mathrm{AU}$ \\
\hline \multicolumn{4}{|l|}{ Viperidae } \\
\hline \multicolumn{4}{|l|}{ Crotalinae } \\
\hline Bothrops alternatus Duméril, Bibron \& Duméril, 1854 & $\mathrm{C}$ & Campo e lavoura & $\mathrm{CA}, \mathrm{AU}$ \\
\hline Bothrops neuwiedii Wagler, 1824 & ZUFSM & Mata e campo & $\mathrm{CA}, \mathrm{M}$ \\
\hline \multicolumn{4}{|l|}{ CROCODILIA } \\
\hline Alligatoridae & & & \\
\hline Caiman latirostris (Daudin, 1802) & $\mathrm{C}$ & Mata e campo & MA \\
\hline
\end{tabular}


Santos, T.G.; Kopp, K. A.; Spies, M.R.; Trevisan, R. \& Cechin, S. Z. - Biota Neotropica, v5 (n1) - BN02705012005

\begin{tabular}{|c|c|c|c|c|}
\hline & campus UFSM & Taim & Gr PoA & Norte Gr PoA \\
\hline Riqueza de espécies & 36 & 21 & 51 & 53 \\
\hline Similaridade com o campus UFSM & & 0,49 & 0,69 & 0,63 \\
\hline Distância (em km) do campus UFSM & & 389 & 306 & 325 \\
\hline Fisionomia & $\begin{array}{c}\text { Campo } \\
\text { Fragmentos de } \\
\text { silvicultura e de } \\
\text { mata em } \\
\text { regeneração } \\
\text { Capoeira } \\
\text { Área antrópica } \\
\text { (lavoura e área } \\
\text { urbanizada) }\end{array}$ & $\begin{array}{c}\text { Campo } \\
\text { Mata } \\
\text { Banhado } \\
\text { Dunas móveis e fixas }\end{array}$ & $\begin{array}{c}\text { Campo } \\
\text { Mata } \\
\text { Banhado } \\
\text { Área antrópica } \\
\text { (lavoura e área } \\
\text { urbana) }\end{array}$ & $\begin{array}{c}\text { Campo } \\
\text { Mata } \\
\text { Banhado } \\
\text { Área antrópica } \\
\text { (lavoura e área } \\
\text { urbanizada) }\end{array}$ \\
\hline
\end{tabular}

Tabela II - Relação entre a fauna de répteis do campus da UFSM, RS, e a Estação Ecológica do Taim, região da grande Porto Alegre (Gr PoA) e região norte da grande Porto Alegre (Norte Gr PoA).

\section{CONCLUSÃO}

Foram registradas, na região do campus da Universidade Federal de Santa Maria, 36 espécies de répteis distribuídas em 12 famílias. A maioria dessas espécies é conhecida por utilizar predominantemente ambientes campestres no Rio Grande do Sul. A região estudada foi fortemente alterada por ação antrópica, possui pequena extensão e é recoberta predominantemente por campo, logo com menor complexidade estrutural que uma área florestal pouco perturbada. Assim, era esperado o registro de uma pequena riqueza de espécies. Por outro lado, essa fauna representa $32 \%$ das espécies de répteis registradas para o Estado. Desta forma, os resultados aqui obtidos indicam a importância do bioma Campos Sulinos para a biodiversidade de répteis do Estado e constituem uma base inicial de conhecimentos sobre história natural e uso de hábitat, subsidiando futuras ações conservacionistas.

\section{AGRADECIMENTOS}

Aos colegas do Laboratório de Herpetologia pelo auxílio nos registros e aos vigilantes da UFSM pela segurança e solicitude prestada. À Dra Denise de Cerqueira Rossa-Feres (UNESP/SJRP) pela revisão do manuscrito e aos dois revisores anônimos pelas valiosas críticas e sugestões.

\section{REFERÊNCIAS BIBLIOGRÁFICAS}

BOULENGER, G.A. 1886. A Synopsis of the Reptiles and Batrachians of the Province Rio Grande do Sul, Brasil. Ann. Mag. Nat. Hist. 18:423-45.

CECHIN, S.T.Z. 1999. História natural de uma comunidade de serpentes na região da Depressão Central (Santa Maria), Rio Grande do Sul, Brasil. Tese de doutorado, Pontifícia Universidade Católica do Rio Grande do Sul, Porto Alegre.
CECHIN, S.T.Z. 2002. Répteis. In Quarta Colônia: Inventários Técnicos (J. Itaqui, org.). Condesus Quarta Colônia, Santa Maria, p.209-214.

COLWELL, R.K. \& CODDINGTON, J.A. 1994. Estimating terrestrial biodiversity through extrapolation. Philos. Trans. Royal Soc. (Ser. B) 345:101-118.

DIXON, J.R. 1989. A key and checklist to the neotropical snakes genus Liophis with country list and maps. Smithsonian Herpetol. Inf. Service 79:1-28.

DUELLMAN, W.E. 1979. The South American Herpetofauna: A Panoramic View. In Its Origin, Evolution and Dispersal (W. E. Duellman, ed.). Mus. Nat. Hist. Kansas, Monogr. 7, p.61-88.

GOMES, N. \& KRAUSE, L. 1982. Lista preliminar de répteis da estação ecológica do Taim, Rio Grande do Sul. Rev. Bras. Zool. 1(1):71-77.

HADDAD, C.F.B. 1998. Biodiversidade dos anfíbios no Estado de São Paulo. In Biodiversidade do Estado de São Paulo, Brasil: síntese do conhecimento ao final do século XX, 6: Vertebrados (R.M.C. Castro, org.) FAPESP, São Paulo, p.15-26.

HADDAD, C.F.B. \& ABE, A. 1999. Anfíbios e Répteis. In Workshop Mata Atlântica e Campos Sulinos. Conservation International do Brasil. Fundação Biodiversitas, Fundação SOS Mata Atlântica, Instituto de Pesquisas Ecológicas, Secretarias do Meio Ambiente de São Paulo e Minas Gerais. Disponível em: <http://www.conservation.org.br/ma/ rp_anfib.htm $>$. Acesso em: 10/08/2003.

KREBS, C.J. 1999. Ecological Methodology. 2 ed., Addison Wesley Education Publishers, Menlo Park, Califórnia.

LEMA, T.DE. 1971. Análise geográfica dos répteis do Estado do Rio Grande do Sul. Arq. Mus. Nac. Rio de J. 54:61-62. 
LEMA, T.DE. 1994. Lista comentada dos répteis ocorrentes no Rio Grande do Sul, Brasil. Comun. Mus. Cienc. Pucrs Ser. Zool. 7:41-150.

LEMA, T.DE. 2002. Os répteis do Rio Grande do Sul. Atuais e fósseis - biogeografia - ofidismo. EDIPUCRS, Porto Alegre.

LEMA, T.DE \& FABIÁN-BEURMANN, M.E. 1977. Levantamento preliminar dos répteis da região da fronteira Brasil - Uruguai. Iheringia Ser. Zool. 50:61-92.

LEMA, T.DE, FABIÁN-BEURMANN, M.E. \& ARAÚJO, M.L. 1980. Lista de répteis encontrados na região da grande Porto Alegre, Estado do Rio Grande do Sul, Brasil. Iheringia Ser. Zool. 55:27-36.

LEMA, T.DE \& FEREIRA, M.T.S. 1990. Contribuição ao conhecimento dos testudines do Rio Grande do Sul (Brasil). Lista sistemática comentada (Reptilia). Acta Biol. Leopold. 12(1):125-64.

LEMA, T.DE, VIEIRA, M.I. \& ARAÚJO, M.L. 1984. Fauna reptiliana do norte da grande Porto Alegre, Rio Grande do Sul, Brasil. Rev. Bras. Zool. 2(4):203-227.

MARQUES, O.A.V. \& SAZIMA, I. 2004. História natural dos répteis da Estação Ecológica Juréia-Itatins. In Estação Ecológica Juréia-Itatins. Ambiente Físico, Flora e Fauna (O.AV. Marques \& W. Duleba, orgs). Holos, Ribeirão Preto, p.257-277.

MARTINS, M. \& OLIVEIRA, M.E. 1999. Natural History of snakes in forests of the Manaus Region, Central Amazonia, Brazil. Herpetol. Nat. Hist. 6(2):78-150.

PEREIRA, P.R.B., NETTO, L.R.G., BORIN, C.J.A. \& SARTORI, M.G.B. 1989. Contribuição à geografia física do município de Santa Maria: unidades de paisagem. Geogr. Ens. Pesqui. 3:37-68.

PETERS, J.A. \& DONOSO-BARROS, R. 1970. Catalogue of the Neotropical Squamata. II. Lizards and amphisbaenians. US Nat. Mus. Bull. 297:1-293.

PETERS, J.A. \& OREJAS-MIRANDA, B. 1970. Catalogue of the Neotropical Squamata. I. Snakes. US Nat. Mus. Bull. 297:1-347.

RUIBAL, R. 1952. Revisionary studies of some South American Teiidae. Bull. Mus. Comp. Zool. 106:477-529.

VANZOLINI, P.E. 1986. Addenda and corrigenda to the Catalogue of Neotropical Squamata. Smithsonian Herpetol. Inf. Service 70:1-25.

VANZOLINI, P.E. 2002. An aid to the identification of the South American species of Amphisbaena (Squamata, Amphisbaenidae). Pap. Avul. Zool. 42(15):351-362.
Título: Répteis do Campus da Universidade Federal de Santa Maria, RS, Brasil

Autores: Tiago Gomes dos Santos, Katia Alcione Kopp, Marcia Regina Spies, Rafael Trevisan, Sonia Zanini Cechin

Biota Neotropica, Vol. 5 ( número 1): 2005 http://www.biotaneotropica.org.br/v 5n 1/pt/ abstract?inventory+BN02705012005

Recebido: 06/04/2004

Revisado: $17 / 12 / 2004$

Publicado: 01/01/2005

ISSN 1676-0603 